\title{
Interacting brains coming in sync through their minds: an interbrain neurofeedback study
}

\author{
Viktor Müller, ${ }^{1}$ Dionysios Perdikis, ${ }^{1,2}$ Melinda A. Mende, ${ }^{1,3}$ and Ulman Lindenberger ${ }^{1,4,5}$ \\ ${ }^{1}$ Center for Lifespan Psychology, Max Planck Institute for Human Development, Berlin, Germany. ${ }^{2}$ Brain Simulation Section, \\ Department of Neurology, Charité-Universitätsmedizin, Berlin, Germany. ${ }^{3}$ Division of Cognitive Sciences, Department of \\ Psychology, University of Potsdam, Potsdam, Germany. ${ }^{4}$ Max Planck UCL Centre for Computational Psychiatry and Ageing \\ Research, London, United Kingdom. ${ }^{5}$ Max Planck UCL Centre for Computational Psychiatry and Ageing Research, Berlin, \\ Germany
}

Address for correspondence: Viktor Müller, Center for Lifespan Psychology, Max Planck Institute for Human Development, Lentzeallee 94, 14195 Berlin, Germany.vmueller@mpib-berlin.mpg.de

\begin{abstract}
Neurophysiological evidence shows that interpersonal action coordination is accompanied by interbrain synchronization (IBS). However, the functional significance of this association remains unclear. Using two experimental designs, we explored whether IBS is amenable to neurofeedback (NFB). Feedback was provided either as two balls approaching each other (so-called ball design), or as two pendula, each reflecting the oscillatory activity of one of the two participants (so-called pendulum design). The NFB was provided at delta (i.e., $2.5 \mathrm{~Hz}$ ) and theta (i.e., $5 \mathrm{~Hz}$ ) electroencephalography frequencies, and manipulated by enhanced and inverse feedback. We showed that the participants were able to increase IBS by using NFB, especially when it was fed back at the theta frequency. Apart from intra- and interbrain coupling, other oscillatory activities (e.g., power spectral density, peak amplitude, and peak frequency) also changed during the task compared with the rest. Moreover, all the measures showed specific correlations with the subjective postsurvey item scores, reflecting subjective feeling and appraisal. We conclude that the use of IBS for NFB might help in specifying the contribution of IBS to interpersonal action coordination and in providing important information about the neural mechanisms of social interaction and the causal dimension of IBS.
\end{abstract}

Keywords: interpersonal neurofeedback; social interaction; interbrain synchrony; power spectral density; subjective feeling and appraisal

\section{Introduction}

Social interactions are an essential part of human life. Coordinated behavior between two social agents is thought to reflect basic needs and dispositions. ${ }^{1,2}$ Recently, it has been associated with and linked to oscillatory couplings between brains. $^{3,4}$ Therefore, investigating both general brain activity within brains and interbrain coupling has become a topic of research in hyperscanning studies, where two or more participants are recorded simultaneously (see Refs. 5 and 6 for recent reviews). Neurophysiological evidence has shown that brain activity synchronizes in coordinated actions $s^{3,4,7-9}$ and bonding behavior. ${ }^{10,11}$ It has also been suggested that the interbrain coupling in coordinated social interaction is not only caused by similarities in input information and produced output, but reflects, at least in part, neural processes oriented to a temporal adjustment of brain functions and network dynamics. ${ }^{7,8,12,13}$ However, the neural mechanisms that implement interpersonally coordinated behavior and support social interaction remain elusive and far from understanding. ${ }^{14,15}$ The present article aims at overcoming limitations and applying the neurofeedback (NFB) methodology 
in a hyperscanning experiment to test whether the participants are able to synchronize their brains by means of the feedback of neural activity across two brains.

It is well known that humans can train to control their brain activity patterns via NFB. ${ }^{16-18}$ NFB may potentially improve the individual's ability to control brain activity through learned self-regulation when visual or auditory stimuli reflecting electrocortical activity and excitability are presented or fed back. ${ }^{19-22}$ Selection of the optimal NFB features is important for success, for example, feedback type, sensor locations, frequency band, training duration, and so on. For example, alpha and theta enhancement training has opened new avenues for emotional learning and psychological growth ${ }^{23,24}$ as well as for enhancing mentalization. ${ }^{18}$ Typically, training lasts over several days or weeks, but short-term effects are also reported. For example, single-channel alpha enhancement with theta crossover can be achieved in sessions of less than 30 minutes. ${ }^{24}$ Recently, it has been shown that not only spectral power in different frequency bands but also coherence and phase synchronization measures between different electrodes can be used as NFB features. ${ }^{25-29}$ In coherence training, participants are reinforced when correlation or coupling between signals at different brain sites is altered in a desired way.

Meanwhile, brain-computer interfaces (BCIs) and NFB technology are not only used for supporting patients ${ }^{20,22,30-35}$ but also to improve attention and $\mathrm{d}^{21,36-40}$ cognitive performance ${ }^{41-43}$ and as a means of control in robotics and gaming. ${ }^{40,44}$ Egner and Gruzelier ${ }^{45}$ reported an enhancement of music performance under stressful conditions in conservatory students through NFB training to raise the theta-alpha $(5-11 \mathrm{~Hz})$ amplitude. There is also a study on collective NFB in an immersive art environment called "My Virtual Dream." ${ }^{36}$ The authors reported data from 523 participants collected in a single night, whereby 20 participants at a time experienced a two-part interaction in the dome with an immersive audiovisual environment in front of 50 spectators. They defined two NFB performance measures based on the ability of the participants to maintain the desired state: relaxation maintenance obtained by changes in alpha spectral power (alpha performance) and concentration maintenance obtained by changes in beta spectral power (beta performance). It has been shown that relaxation conditions showed a gradual decrease of spectral power in midrange (8-20 $\mathrm{Hz})$ and high $(35-45 \mathrm{~Hz})$ frequencies, whereas concentration training revealed a gradual increase of power in the beta range and a decrease in low frequencies $(<3 \mathrm{~Hz})$. The authors indicated that participants were able to learn to modulate their relative spectral power by NFB within only 60 and 80 $s$ training periods (for relaxation and concentration maintenance, respectively). ${ }^{46}$ Other studies used a crossbrain NFB experimental design with two participants who regulated their near-infrared spectroscopy or electroencephalography (EEG) activity in terms of amplitude differences. ${ }^{47,48}$ None of the studies used interbrain synchronization (IBS) as an NFB feature to regulate the interaction of the minds, with the exception of an NFB study on three pigeons. ${ }^{49}$ In summary, positive results regarding the intentionally induced or enhanced IBS by means of NFB will provide evidence that IBS is more than just an epiphenomenon of the sensorimotor response and that socially adapted behavior can be altered when a delicate oscillatory balance between the agents is achieved or changed (learned). It should also indicate that IBS does causally facilitate social interaction. ${ }^{50}$ Whether (and if so, how) NFB can influence IBS or whether IBS can be used as an NFB feature in a hyperbrain BCI setting is completely unknown.

The present study was designed to fill this knowledge gap and find out whether participants can synchronize their brains using NFB. For these purposes, we developed two different NFB experimental designs that reflect interbrain synchronous EEG activity and fed it back visually: (1) the socalled ball design, reflecting the common interbrain state of two participants, where the in-phase synchrony between two participants' frontocentral electrode sites, measured by the absolute coupling index (ACI), ${ }^{8,51}$ is fed back as two balls approaching each other, and (2) the so-called pendulum design, where the oscillatory activity (instantaneous phase angles) of two participants (also measured frontocentrally) is fed back in the form of two pendulums. Thus, in the former case, the participants were able to only control the common IBS state and, in the latter, they were each able to control their own oscillatory brain state (pendulum) and adjust both of them to each other. We used a post-questionnaire to 
evaluate subjective feeling, test partner's likability, and estimated capability to influence the task. We correlated these items with EEG outcomes, to provide further subjective information about synchronized states. The expectation was that several EEG outcomes could be beneficial for the task and be associated with a positive feeling and test partner's likability.

\section{Methods}

\section{Participants}

All participants had normal or corrected-to-normal vision and did not suffer from any psychological, developmental, or neurological disorder. The gender of the participants was balanced so that an equal number of female-female, female-male, and malemale pairs was tested, as it has been found that differences in gender composition of the dyads can influence patterns of interbrain connectivity (e.g., Ref. 52). Initially, 27 dyads (9 female-female, 9 female-male, and 9 male-male) participated in the current study. Data of two of the dyads (one femalemale and one male-male) were excluded from the final analysis due to technical issues. Thus, the sample that was included into the final analysis consisted of 25 dyads ( 9 female-female, 8 female-male, and 8 male-male; mean age $=26.8$ years, $\mathrm{SD}=$ 3.1 years). Neither member of each dyad knew one another before the testing. All participants provided written informed consent prior to the study. The study was approved by the Ethics Committee of the Max Planck Society and was conducted according to the ethical standards stated in the 1964 Declaration of Helsinki.

\section{Psychological assessment}

Immediately before testing, participants were asked to fill in a consumption questionnaire about factors influencing the EEG experiment, for instance, consumption of caffeine and alcohol. In addition, educational background was assessed for each participant. After testing, participants filled in two questionnaires while still sitting in the EEG cabin. These were a post-questionnaire about the experiment and a likeability questionnaire. ${ }^{53}$ The likeability questionnaire consisted of 11 equally pooled items that were transformed to a single likeability scale through averaging across the items. Together with the post-questionnaire, there were overall 16 items evaluating subjective feeling, test partner's lik- ability, and estimated capability to influence the task using different strategies, such as concentration, relaxation, generation of thoughts, and mental calculation (Table S1, online only). The scores of these items were used for correlation analyses, in order to provide further information about synchronized states.

\section{Procedure}

During the testing sessions, the dyads were asked to sit back-to-back in an EEG cabin that was electromagnetically and acoustically shielded. This setup was chosen to prevent participants from communicating with each other or coordinating their movements. Rather, they sat in the EEG cabin and interacted with each other only by knowing that they were performing the NFB task together. The experiment consisted of 2 resting state and 24 NFB task sessions. The first session was a prerest condition to record a baseline of the brain oscillations of each participant. In this session, participants were asked to sit calmly and relaxed in front of the computer screen without moving their bodies, specifically their limbs, tongue, chin, eyes, and head. The rest condition lasted for 4 minutes. Participants sat 2 min with opened eyes and another 2 min with eyes closed. After the relaxation period, the participants performed different NFB tasks: delta and theta ball tasks as well as delta and theta pendulum tasks. The last session was another resting state session and consisted of the same procedure as the resting session at the beginning of the experiment. The design of the task was blockwise, meaning that it either started with a block of ball trials or pendulum trials (Fig. 1A). Within the blocks, there were two subblocks based on the calculation of the IBS and corresponding feedback either in the delta $(2.5 \mathrm{~Hz})$ or the theta $(5.0 \mathrm{~Hz})$ frequency. Within these subblocks, there were six trials, always in the same order. The block started with two normal NFB trials, followed by a fake trial with enhanced feedback. The fourth and fifth trials were again normal trials followed by a final inverted trial. We used a fixed trial order, where the fake trials were always placed in the middle of the normal NFB trials and the inverted trials come up at the end of each task condition, to avoid a possible frustrating effect of inverted trials on the trials that immediately followed (Fig. 1A). This block design led to eight different possible experimental orders that were matched 
A

Experimental design

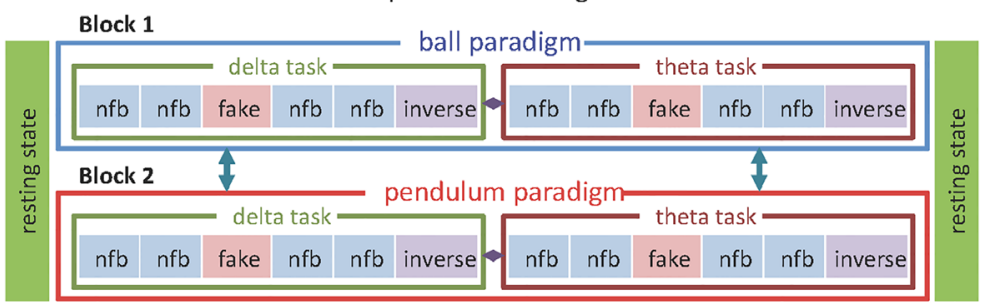

B

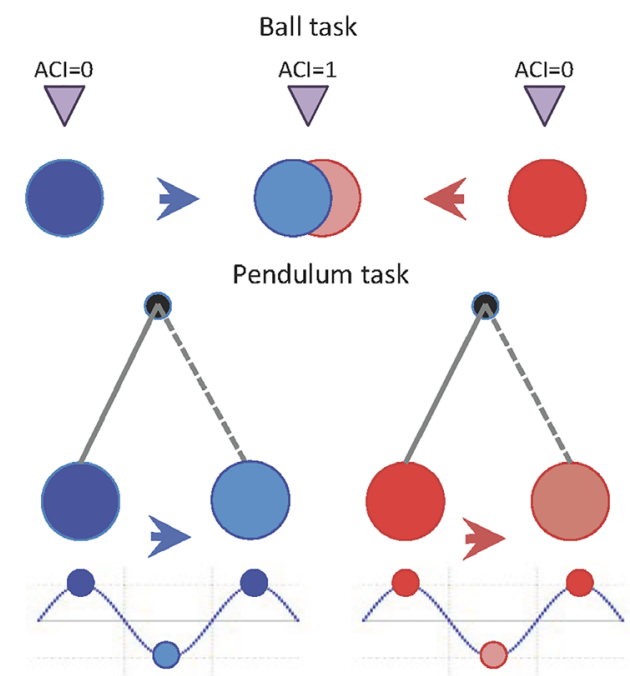

C

Calculation of the Absolute Coupling Index $(\mathrm{ACl})$

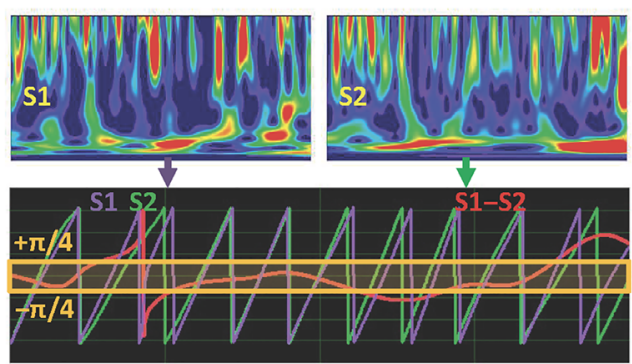

Figure 1. Experimental design, NFB task paradigms, and synchronization measure. (A) Experimental design. The experiment consisted of 2 resting state and 24 NFB task sessions. The 24 NFB task sessions were divided into two blocks of 12 sessions each. Each block consisted either of ball or pendulum task conditions, which were divided in two subblocks with delta or theta NFB tasks. If the blocks and subblocks were randomized across participants (indicated by arrows), then the single NFB tasks were always presented in the same order as displayed in the picture. (B) Ball and pendulum task paradigms. In the ball task (on the top), the balls moved toward or away from each other depending on the IBS measured by the absolute coupling index (ACI; as presented in panel C) at frontocentral electrodes averaged beforehand for each participant's brain. When the ACI is high, the balls tend to overlap each other; when the ACI is low, the balls move to the outer borders of the screen. In the pendulum task paradigm (on the bottom), each of the two pendulums represented the brain oscillations or instantaneous phase angles (also at frontocentral sites) of each of the participants. They were asked to make them swing in-phase, so that pendulum movements were parallel to each other. (C) Calculation of the ACI. For calculation of the ACI, a complex Gabor wavelet time-frequency transform was computed on the signals of the two participants' brains (S1 and S2) in order to extract the instantaneous phases of them and to calculate the instantaneous phase difference (S1-S2) at the corresponding task frequency. All the phase difference points (in red) lying in the range between $-\pi / 4$ and $+\pi / 4$ (yellow area) were summed up and divided by the overall number of (red) points. Thus, the ACI was normalized between 0 (no synchronization) and 1 (absolute in-phase synchronization). 
with relation to female-female, female-male, and male-male dyads. In total, each dyad performed 24 trials lasting $210 \mathrm{~s}$ each. Participants took a short break between every 5-10 trials so that the duration of the experiment varied between 70 and 90 minutes.

Before the experiment, the participants were instructed as follows:

\begin{abstract}
"Your task will be (1) to move the two balls towards each other or (2) to make the two pendulums swing synchronously in the same rhythm, controlling your brain potentials accordingly. In the first task, the balls will only move towards each other when you are synchronized with your partner; in the second task, you are each responsible for your own pendulum. Different strategies can be used for controlling your brain waves, such as relaxation, activation, generating thoughts, mental calculation, etc. You are free to figure out how to control your brain waves. It might not work out, but it is worth a try for you, as it can help you learn to control and manage your activation state also in relation to other people."
\end{abstract}

\section{Dyadic NFB paradigms}

During the NFB tasks, participants were asked to look at the computer screen with an NFB visualization, which consisted of either two balls with different colors (red and blue) or two pendulums also with two different colors (red and blue). In the ball condition, the balls moved toward or away from each other in the horizontal plane in the middle of the screen. When the IBS measured by the $\mathrm{ACI}^{8,51}$ was high, the balls tended to overlap each other; when the ACI was low, the balls moved to the outer borders of the screen (Fig. 1B). Thus, the task in this condition was to move the balls as close as possible to each other and to make them visually overlapped. In the pendulum condition, each of the two pendulums represented the brain oscillations (instantaneous phase angles) of each of the participants. They were asked to make them swing in phase, so that the pendulum movements were parallel to each other (Fig. 1B). Thus, in this task, each participant was able to control their own pendulum to achieve in-phase synchrony. In the ball task, we used the ACI measure, as it evaluates the strictest sense of in-phase synchronization as a stable and small phase difference or time coincidence of two oscillations. It reflects the ratio of time points of the phase difference lying within the narrow interval $[-\pi / 4,+\pi / 4]$ to the total number of points in the whole interval (Fig. 1C).
As mentioned before, the study contained three different NFB conditions (normal, enhanced, and inverted feedback). In the normal condition, NFB was normally visualized on the screens to give participants an impression of their actual performance. In the trials with enhanced feedback (fake condition), the visualization of NFB was improved to motivate the dyads by giving them the impression that they were performing well in the experiment. In the trials with inverted feedback, the visual feedback improved when the brain oscillations of the dyad were in antiphase or desynchronized (negative learning). These trials were implemented as a control condition to examine whether performance was as good as in normal trials. Participants did not receive any instructions concerning the different experimental conditions (normal, enhanced, or inverted feedback) so that they were blind to the testing conditions (the workflow of the different trials can be viewed in the Supplementary Movies, online only).

\section{EEG recordings and offline analyses}

EEGs were recorded from the dyads with 64 active $\mathrm{Ag} / \mathrm{AgCl}$ electrodes per person at a sampling rate of $1000 \mathrm{~Hz}$, with the reference electrode placed at the right mastoid (actiCAP, Brain Products, Munich, Germany). Recorded frequency bands ranged from 0.01 to $250 \mathrm{~Hz}$. The EEG caps were placed on the scalp according to the international 10-10 system. In addition, an electrooculogram in vertical and horizontal dimensions was obtained from each participant to control for eye movement. Each participant was recorded with a separate amplifier with separate grounds that were coupled to the same computer. In addition to the EEG measure, heart rate, galvanic skin response, and breathing rate were recorded during the testing. Note that we report only hyperscanning EEG data in this paper. For offline analyses, EEG recordings were rereferenced to an average of the left and right mastoid separately for each subject and filtered with a band pass ranging from 0.5 to $100 \mathrm{~Hz}$. The notch filter was set to $50 \mathrm{~Hz}$. Eye movement correction was accomplished by independent component analysis. ${ }^{54}$ For spectral power analyses, the EEGs were segmented into epochs of 4096 data points with 50\% overlap. The power spectral density (PSD) was calculated using Welch's method and the Hanning window function in the four frequency ranges: delta (0.5- 
$4 \mathrm{~Hz})$, theta $(4-8 \mathrm{~Hz})$, alpha $(8-14 \mathrm{~Hz})$, and beta (14-28 Hz). Within these frequency ranges, the peak amplitude and peak frequency were determined. Intra- and interbrain synchronization were assessed using the ACI measure ${ }^{8,51}$ for all possible electrode pairs within and between the brains, respectively. The ACI reflecting in-phase synchronization was determined for four frequency bins $(2.5,5,10$, and $20 \mathrm{~Hz})$ within the epochs of $5 \mathrm{~s}$ without overlap. For each electrode location, ACI coupling strengths were calculated as a sum of all coupling pairs from one electrode to all others separately for within- and between-brain coupling. For statistical analyses, all the measures were averaged across the epochs.

\section{EEG real-time feedback generation}

The NFB was calculated online using six frontocentral channels from each participant (F3, Fz, F4, C3, $\mathrm{Cz}$, and $\mathrm{C} 4$ ). Our choice for these electrodes was based on the results of hyperscanning studies, which showed that these brain regions play a crucial role in tasks involving interpersonal interaction. ${ }^{4,8,11,13}$ Signals from these electrodes were averaged within the two brains to create a single feedback time series for each participant $(i): \overline{E E G}_{i}(t)$. These time series were determined for the time segment of $4 \mathrm{~s}$ centered around time $t$. The complex Gabor wavelet timefrequency transform was computed on $\overline{E E G}_{i}(t)$ in order to extract the unwrapped instantaneous phase of each participant $\varphi_{i}(t)$, which was used in a different way for each of the two experimental designs. The feedback EEG signal was sent in packages of $T_{s}=17 \mathrm{~ms}$, which was the time resolution of the feedback. The packages were gathered into a buffer of $4 \mathrm{~s}$ and we further processed such time segments (i.e., of the last $4 \mathrm{~s}$ ) to generate the feedback signal. The display was refreshed approximately for every new feedback data package, since its refresh rate was $60 \mathrm{~Hz}$, resulting in a refresh time of $17 \mathrm{~ms}$ (see Supplementary Materials, online only, for details).

The whole experiment was performed in MATLAB 2012b (the MathWorks, Inc., Natick, MA) code (including the statistics toolbox and psychtoolbox) written by the authors. For the real-time connection of the recording computer to the experimental stimulus computer and vice versa, we used scripts provided by Brain Products GmbH (Gilching, Germany).

\section{Statistical analyses}

For statistical analyses, the EEG spectral measures (PSD, peak amplitude, and peak frequency) and ACI coupling strengths (sum of all coupling pairs from one electrode to all others) were first determined for 60 electrode locations and then collapsed into nine brain sites: FL (frontal left), FZ (midfrontal), FR (frontal right), CL (central left), CZ (mid-central), CR (central right), PL (parietal left), PZ (mid-parietal), and PR (parietal right). All the measures were analyzed using three-way repeated measures ANOVAs with the within-subject factors condition (rest, nfb, fake, and inverse task conditions), anteroposterior ( $\mathrm{F}=$ frontal, $\mathrm{C}=$ central, and $\mathrm{P}=$ parietal), and mediolateral $(\mathrm{L}=\mathrm{left}, \mathrm{Z}=\mathrm{mid}$, and $\mathrm{R}=$ right). To investigate how stable the peak frequencies were within the pairs, we determined the peak frequency difference changes within the test pairs and subjected them to the ANOVA as before. The four postsurvey items, reflecting estimated capability to influence the task, were analyzed using two-way repeated measures ANOVA with the two within-subject factors items (4 items) and task (ball versus pendulum). Greenhouse-Geisser epsilons were used in all ANOVAs for a nonsphericity correction when necessary. The StudentNewman-Keuls (SNK) test was employed for post hoc testing. To relate the EEG indices to behavioral measures, we correlated the former with the 16 items of the postsurvey that evaluated subjective feeling, test partner's likability, and estimated capability to influence the task. Spearman's rank correlation was used for this purpose.

\section{Results}

\section{NFB performance}

To better understand changes in EEG dynamics, we first present NFB task performance since this was shown during the experiment on the screen. Note that this performance in fake and inverse task conditions results from the corresponding manipulations as described in the Methods. In other words, this performance indicates results of the feedback applied during the experiment. Examples of different task conditions are shown in the Supplementary Movies (online only). The task performance was measured as the time of the balls' coincidence in the ball task and as the time of being in-phase in the pendulum task. The NFB task performance was calculated at five different threshold levels. The 
A

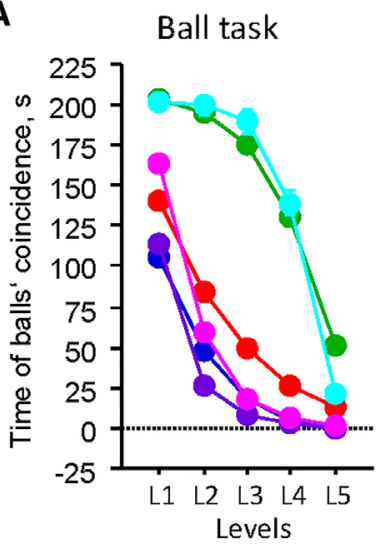

\section{B \\ B Pendulum task}

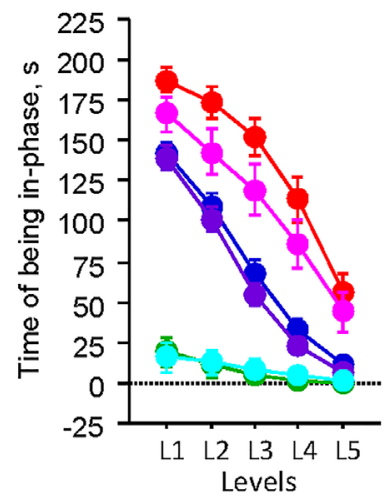

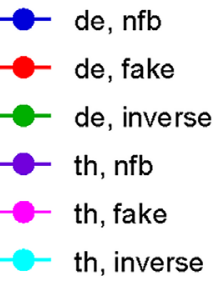

C

Capability to control the ball or the pendulum

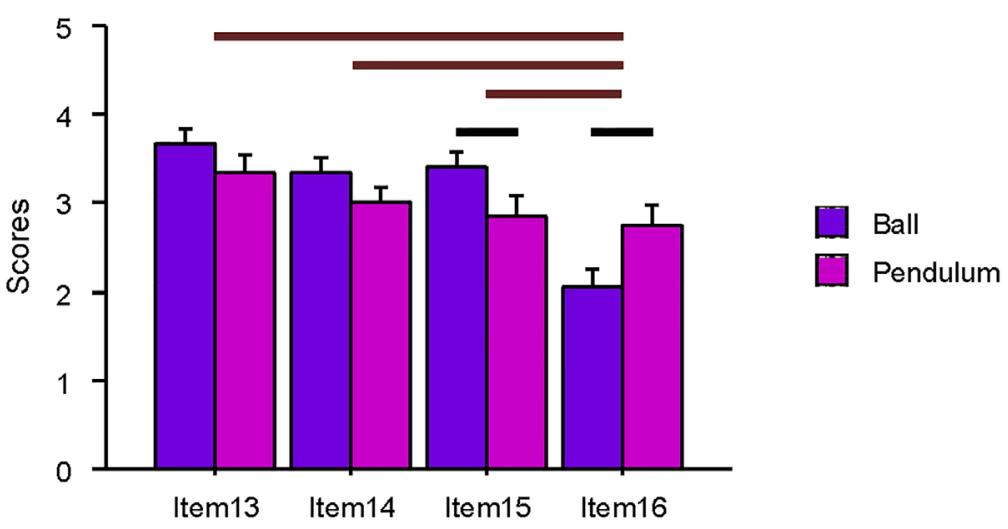

Figure 2. NFB performance and postsurvey scores of the capability to control the ball or the pendulum. (A) NFB performance in the ball task. The NFB performance in this task was measured as time (in seconds) of the balls' coincidence at five different threshold levels corresponding to synchronization levels measured by the ACI: L1 (ACI > 0.5); L2 (ACI > 0.6); L3 (ACI > 0.7); L4 (ACI > 0.8); and L5 (ACI > 0.9). (B) NFB performance in the pendulum task. The NFB performance in this task was measured as time (in seconds) of being in-phase of the two pendula controlled by the two participants. It was also calculated at five different threshold levels dependent on absolute phase difference (aPD): L1 (aPD < 0.5 ); L2 (aPD < $0.4 \pi)$; L3 (aPD < $0.3 \pi)$; L4 (aPD $<0.2 \pi)$; and L5 (aPD < $0.1 \pi)$. (C) Postsurvey scores of the capability to control the ball or the pendulum task. Bar diagrams represent means and standard errors across the four items indicating estimated capability to influence the ball or pendulum task using different strategies, such as concentration (item 13), relaxation (item 14), generation of thoughts (item 15), and mental calculation (item 16). The horizontal lines indicate significant differences $(P<0.05)$ between the items (brown) or tasks (black).

threshold levels indicated synchronization levels measured by the ACI (ball task) or phase difference (pendulum task). In the former case, the threshold levels correspond to synchronization levels of: L1 (ACI >0.5); L2 (ACI >0.6); L3 (ACI >0.7); L4 (ACI >0.8); and L5 (ACI >0.9). In the latter case, the threshold level was calculated depending on the absolute phase difference (aPD): L1 ( $\mathrm{aPD}<0.5 \pi$ ); $\mathrm{L} 2(\mathrm{aPD}<0.4 \pi) ; \mathrm{L} 3(\mathrm{aPD}<0.3 \pi) ; \mathrm{L} 4(\mathrm{aPD}<0.2 \pi)$; and L5 $(\mathrm{aPD}<0.1 \pi)$. Results of this analysis are presented in Figure 2A and B. It can be seen that perfor- mance declined exponentially with the increasing synchronization threshold. In the ball task, the highest performance (time of balls' coincidence in seconds) was in the inverse task condition because the lowest synchronization was transformed in this task condition to its inverse, that is, the balls overlapped for most of the time (high coincidence time). This corresponds then to negative learning. The next best performance was achieved during the fake condition, because the performance shown during the task (real feedback) was improved by the 
improvement factor (see Supplementary Materials for details, online only). The lowest performance was achieved during the normal NFB task condition. In the pendulum task, the performance (time of being in phase in seconds) was the highest in the fake task condition, also due to the improvement of the feedback through the improvement factor, and the lowest in the inverse task condition. The latter probably happened because the movement of the pendulums always began in antiphase and it was difficult for the participants to get them in sync. Contrary to the ball task with negative learning in the inverse condition, we observed in the pendulum task a negative feedback that frustrated the participants through an apparent inefficiency to control the pendula or to improve their performance.

\section{Postsurvey item scores}

The mean and standard deviation of the postsurvey item scores are presented in Table S1 (online only). First, the participants reported being more tired at the end of the experiment than at the beginning. This difference was highly significant $(t(49)=15.0$, $P<0.0001)$. Second, estimated capability to influence the task using different strategies, such as concentration, relaxation, generation of thoughts, and mental calculation, was different with regard to the ball and pendulum tasks, at least for the generation of thoughts and mental calculation. We analyzed the four last items using two-way repeated measures ANOVA with the two within-subject factors items (4 items) and task (ball versus pendulum). The ANOVA revealed a significant main effect of the factor items $(F(6,294)=12.71, P<0.0001$, $\left.\eta^{2}=0.21\right)$ and a significant interaction of items $\times$ $\operatorname{task}\left(F(6,294)=6.34, P<0.001, \eta^{2}=0.12\right)$. As displayed in Figure 2C, mental calculation is the least used strategy as compared with the other three strategies. But interestingly, this strategy (i.e., mental calculation) was used significantly more in the pendulum task $(t(49)=3.30, P<0.018)$, and the generation of thoughts was used significantly more in the ball task $(t(49)=2.59, P<0.013)$.

\section{Power spectral density}

Before describing phase synchronization changes under different NFB conditions, we first present PSD changes to show how, overall, the oscillatory brain activity is evolving or changing under these conditions. For these purposes, we concentrate on the frequencies of interest manipulated during the task (i.e., delta and theta) and also describe other frequencies (e.g., alpha and beta) to better understand the effects.

Corresponding log-transformed PSD changes were analyzed using three-way repeated measures ANOVAs (condition $\times$ anteroposterior $\times$ mediolateral). Results of these ANOVAs for the four frequency bands restricted to the factor condition and interactions with this factor are presented in Table 1 and Figure 3 (see also Table S2 and Fig. S1, online only, for details). It can be seen that the main effect condition and the interaction condition $\times$ anteroposterior were significant in practically all four frequency bands, with the exception of the condition $x$ anteroposterior interaction in the delta pendulum task. The SNK post hoc test revealed a significant decrease of PSD in the NFB tasks compared with the rest condition in all four frequency bands (Fig. 3). This decrease of PSD in task conditions compared with the rest takes place to a greater extent at parietal electrode sites.

\section{Power spectral peak amplitude and peak frequency}

We further investigated the power spectral peak amplitude and peak frequency during the task and rest conditions. Whereas the peak amplitude changes were relatively similar to the PSD changes (Table S3 and Fig. S2, online only), the peak frequency revealed a significant main effect of condition in the delta frequency band during the pendulum tasks and in the theta frequency band among all NFB tasks. As shown in Figure 4, delta (in the pendulum tasks) and theta peak frequencies decrease during the NFB tasks compared with the rest condition. Whereas the delta peak frequency, distributed around $1 \mathrm{~Hz}$, was far from the delta task frequency (i.e., $2.5 \mathrm{~Hz}$ ), the theta peak frequency approached from about $6 \mathrm{~Hz}$ during the rest condition to the frequency of about $5.5 \mathrm{~Hz}$ during the task conditions, which is relatively close to the theta task frequency (i.e., $5 \mathrm{~Hz}$ ). Most interestingly, we analyzed the peak frequency differences within the pairs. The task frequencies, that is, delta and theta peak frequencies, did not show any significant changes across conditions, whereas alpha and beta peak frequencies revealed a significant main effect of the factor condition in all four tasks, with the exception of nonsignificant differences in the alpha peak frequency during the delta ball task. Significant interactions 
A

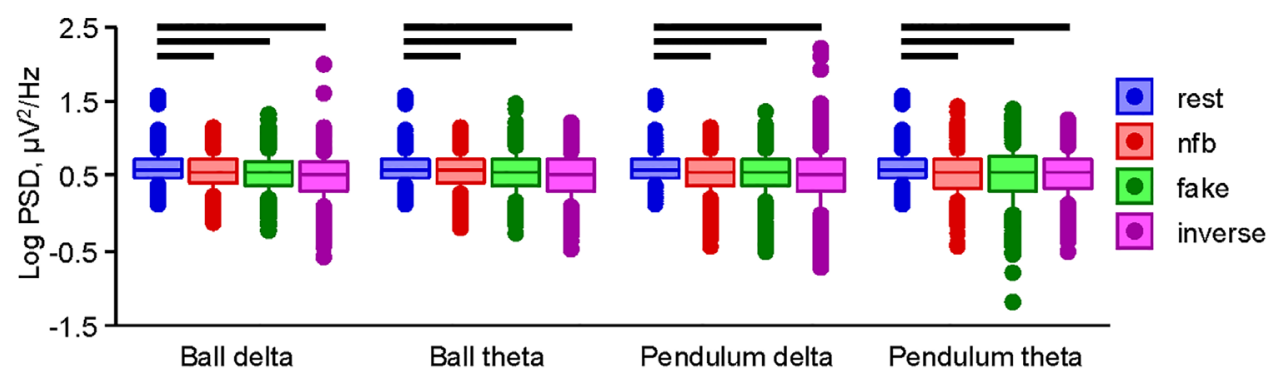

B

Theta PSD

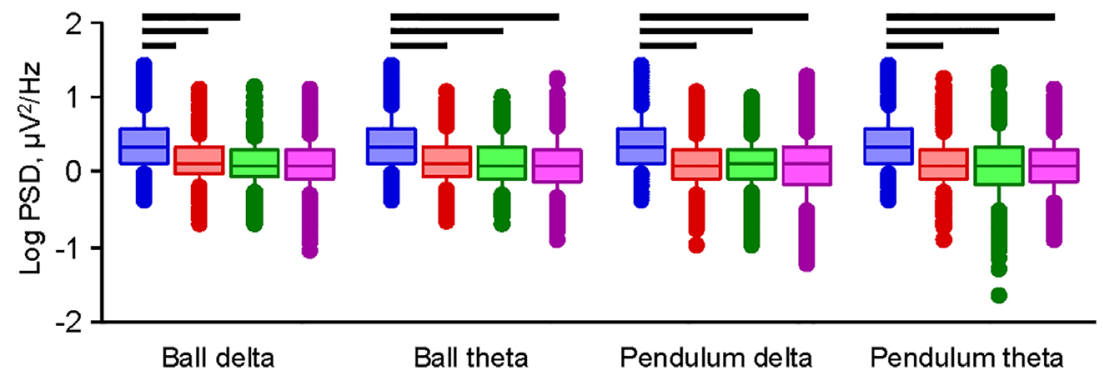

C

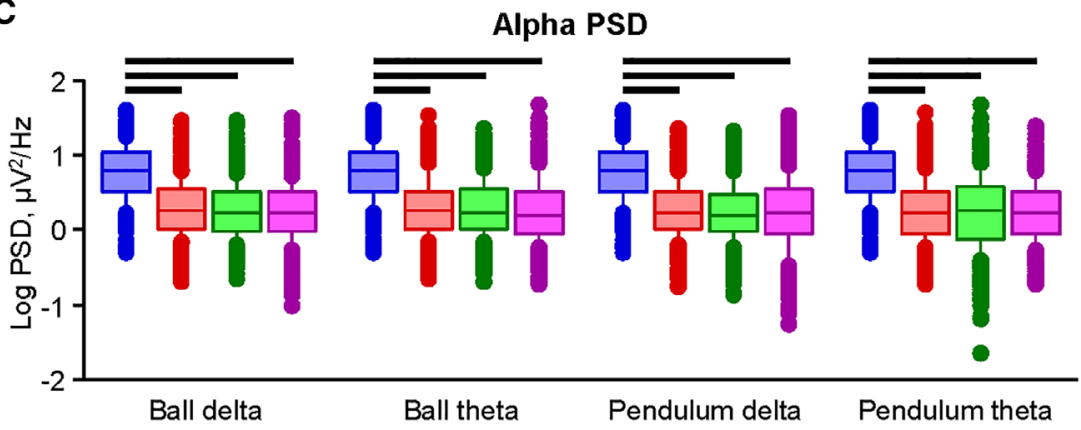

D

Beta PSD

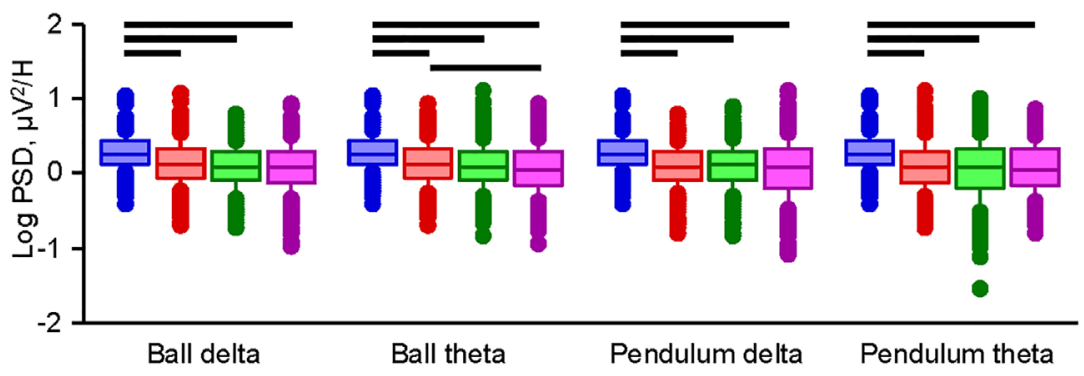

Figure 3. Box plots of the PSD changes across the task conditions in the four frequency bands. (A) Delta PSD across the four NFB tasks and the four task conditions. (B) Theta PSD across the four NFB tasks and the four task conditions. (C) Alpha PSD across the four NFB tasks and the four task conditions. (D) Beta PSD across the four NFB tasks and the four task conditions. Box plots represent PSD values across the four tasks (ball delta, ball theta, pendulum delta, and pendulum theta) and the four task conditions: resting state, normal NFB, fake, and inverse task conditions. Horizontal bold lines indicate significant differences as revealed by the SNK post-hoc test $(P<0.05)$. 
Table 1. ANOVA results for the PSD and the peak frequency across the four frequency bands in the four NFB tasks

\begin{tabular}{|c|c|c|c|c|c|c|c|c|c|c|c|c|}
\hline & \multicolumn{6}{|c|}{ Ball design } & \multicolumn{6}{|c|}{ Pendulum design } \\
\hline & \multicolumn{3}{|c|}{ Delta } & \multicolumn{3}{|c|}{ Theta } & \multicolumn{3}{|c|}{ Delta } & \multicolumn{3}{|c|}{ Theta } \\
\hline & $F$ & $P$ & $\eta^{2}$ & $F$ & $P$ & $\eta^{2}$ & $F$ & $P$ & $\eta^{2}$ & $F$ & $P$ & $\eta^{2}$ \\
\hline \multicolumn{13}{|l|}{ Delta PSD } \\
\hline $\mathrm{C}$ & 5.99 & 0.004 & 0.11 & 6.45 & 0.004 & 0.12 & 4.35 & 0.018 & 0.082 & 4.18 & 0.019 & 0.079 \\
\hline $\mathrm{C} \times \mathrm{AP}$ & 2.88 & 0.042 & 0.056 & 2.70 & 0.043 & 0.052 & 2.27 & 0.077 & 0.044 & 2.33 & 0.075 & 0.045 \\
\hline \multicolumn{13}{|l|}{ Theta PSD } \\
\hline $\mathrm{C}$ & 26.86 & 0.000 & 0.35 & 25.95 & 0.000 & 0.35 & 25.57 & 0.000 & 0.34 & 24.27 & 0.000 & 0.33 \\
\hline $\mathrm{C} \times \mathrm{AP}$ & 6.71 & 0.000 & 0.12 & 7.56 & 0.000 & 0.13 & 3.78 & 0.011 & 0.072 & 5.82 & 0.001 & 0.11 \\
\hline \multicolumn{13}{|l|}{ Alpha PSD } \\
\hline C & 100.66 & 0.000 & 0.67 & 89.54 & 0.000 & 0.65 & 72.82 & 0.000 & 0.60 & 67.22 & 0.000 & 0.58 \\
\hline $\mathrm{C} \times \mathrm{AP}$ & 15.99 & 0.000 & 0.25 & 18.94 & 0.000 & 0.28 & 15.51 & 0.000 & 0.24 & 15.46 & 0.000 & 0.24 \\
\hline \multicolumn{13}{|l|}{ Beta PSD } \\
\hline $\mathrm{C}$ & 22.06 & 0.000 & 0.31 & 27.68 & 0.000 & 0.36 & 20.07 & 0.000 & 0.29 & 19.71 & 0.000 & 0.29 \\
\hline $\mathrm{C} \times \mathrm{AP}$ & 8.28 & 0.000 & 0.15 & 8.98 & 0.000 & 0.16 & 10.68 & 0.000 & 0.18 & 8.94 & 0.001 & 0.15 \\
\hline \multicolumn{13}{|c|}{ Delta peak frequency } \\
\hline $\mathrm{C}$ & 1.20 & 0.31 & 0.024 & 2.54 & 0.086 & 0.049 & 7.10 & 0.001 & 0.13 & 11.69 & 0.000 & 0.19 \\
\hline $\mathrm{C} \times \mathrm{AP}$ & 0.61 & 0.65 & 0.012 & 0.42 & 0.77 & 0.008 & 1.54 & 0.20 & 0.030 & 1.12 & 0.35 & 0.022 \\
\hline \multicolumn{13}{|c|}{ Theta peak frequency } \\
\hline $\mathrm{C}$ & 40.23 & 0.000 & 0.45 & 42.14 & 0.000 & 0.46 & 34.18 & 0.000 & 0.41 & 30.94 & 0.000 & 0.39 \\
\hline $\mathrm{C} \times \mathrm{AP}$ & 1.25 & 0.29 & 0.025 & 0.54 & 0.65 & 0.011 & 2.35 & 0.074 & 0.046 & 0.66 & 0.60 & 0.013 \\
\hline \multicolumn{13}{|c|}{ Alpha peak frequency } \\
\hline $\mathrm{C} \times \mathrm{ML}$ & 4.47 & 0.001 & 0.084 & 4.61 & 0.001 & 0.086 & 3.84 & 0.005 & 0.073 & 4.28 & 0.003 & 0.080 \\
\hline $\mathrm{C} \times \mathrm{AP} \times \mathrm{ML}$ & 2.63 & 0.014 & 0.051 & 4.86 & 0.000 & 0.090 & 1.93 & 0.065 & 0.038 & 0.81 & 0.57 & 0.016 \\
\hline \multicolumn{13}{|c|}{ Beta peak frequency } \\
\hline $\mathrm{C} \times \mathrm{AP}$ & 6.35 & 0.001 & 0.12 & 7.90 & 0.000 & 0.14 & 5.71 & 0.001 & 0.10 & 6.80 & 0.000 & 0.12 \\
\hline $\mathrm{C} \times \mathrm{ML}$ & 2.61 & 0.027 & 0.051 & 1.31 & 0.26 & 0.026 & 2.88 & 0.024 & 0.056 & 1.39 & 0.24 & 0.027 \\
\hline
\end{tabular}

Bold values indicate $P<0.05$; AP, anteroposterior; C, condition; ML, mediolateral.

for alpha and beta peak frequencies as well as significant peak frequency differences within the participant pairs at these two frequencies are presented in the Supplementary Material (online only).

\section{Correlations of PSD and peak frequency with postsurvey item scores}

We further correlated PSD and peak frequencies during the NFB task condition with the 16 postsurvey item scores reflecting subjective feeling, test partner's likability, and estimated capability to influence the task (Table S1, online only). The results of these correlation analyses are presented in Figure 5. There were relatively strong positive correlations of PSD in the theta, alpha, and beta frequency bands with tiredness at the end of the experiment (item 2) and nervousness (item 3); delta PSD correlated positively with tiredness at the end of the experiment and expectation of an important appointment (item 4). Note that there were no significant correlations with tiredness at the beginning of the experiment (item 1). In all frequency bands, PSD correlated negatively with the sympathy for the test partner (item 8). In addition, there were positive correlations between delta PSD (during the ball task) and estimated capability to influence the task through relaxation (item 14) as well as also negative correlations of theta, alpha, and beta PSD with some other scores of the estimated capability to influence the task (Fig. 5).

Regarding the peak frequencies, there was a relatively strong positive correlation of the theta peak frequency with nervousness (item 3), and negative correlations of the theta peak frequency with patience during experiment (item 6) and with estimated capability to influence the task through concentration and thoughts (items 13 and 15, respectively). The alpha peak frequency correlated 


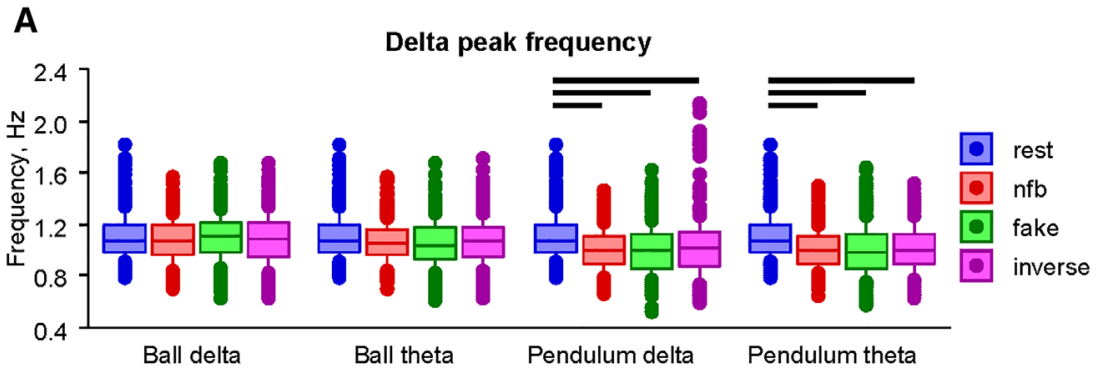

B

Theta peak frequency

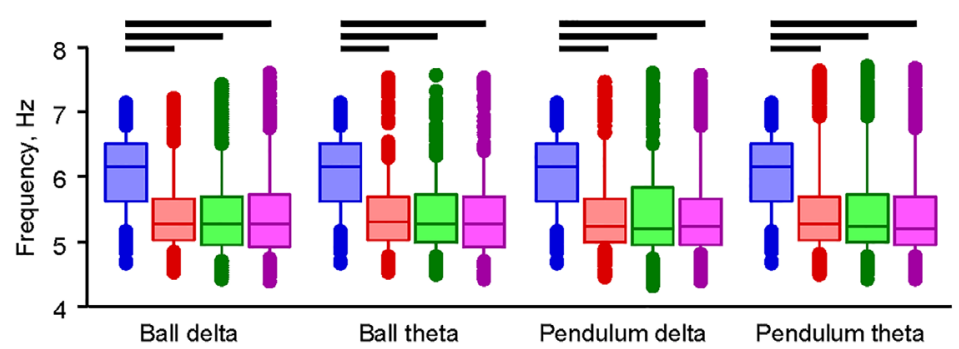

Figure 4. Box plots of the peak frequency changes across the task conditions in the delta and theta frequency bands. (A) Delta peak frequency across the four NFB tasks and the four task conditions. (B) The theta peak frequency across the four NFB tasks and the four task conditions. Box plots represent peak frequency values across the four tasks (ball delta, ball theta, pendulum delta, and pendulum theta) and the four task conditions: resting state, normal NFB, fake, and inverse task conditions. Horizontal bold lines indicate significant differences as revealed by the SNK post-hoc test $(P<0.05)$.

mostly negatively, especially with general patience (item 5) and estimated capability to influence the task through concentration and, to some extent, through mental calculations (items 13 and 16, respectively) during the ball task. Interestingly, during the pendulum task, this correlation was positive at frontocentral sites, when correlated with the capability to influence the task through concentration, and negative at parietal sites, when correlated with the estimated capability to influence the task through relaxation. The beta peak frequency correlated strongly negatively with tiredness at the end of the experiment (item 2). In addition, there were some positive correlation between the beta peak frequency during the ball task and the estimated capability to influence the task through concentration (item 13).

\section{Intra- and interbrain synchrony changes}

Unlike with the power spectral indices, the ACI measures were determined for individual frequency bins (i.e., 2.5, 5, 10, and $20 \mathrm{~Hz}$ ). Although the $\mathrm{ACI}$ is a symmetric measure, the topological distribution of interbrain strengths in the two brains averaged across the nine brain sites was different. Hence, we determined the average strength for each participant within and between the brains and subjected them to three-way ANOVAs (condition $\times$ anteroposterior $\times$ mediolateral $)$ similar to the analyses of spectral indices described above. The results of the within- and between-brain coupling for the four frequency bins are presented in Table 2 and Tables S6 and S7 (online only). It can be seen that the main effect of the factor condition for the intrabrain coupling strength for delta and theta frequency bins was significant in the theta pendulum task only. The intrabrain strengths in the fake condition decreased significantly compared with the rest and inverse task condition (Fig. S4, online only). The intrabrain alpha strengths revealed a significant main effect of condition in all NFB tasks with the exception of the delta pendulum task, and the intrabrain beta strengths revealed a significant main effect of condition in the theta ball and theta pendulum tasks. In addition, intrabrain strength in all four frequencies revealed a significant interaction of condition $\times$ anteroposterior among all the four NFB tasks (Table 2). As shown in 
A

Delta PSD

Theta PSD
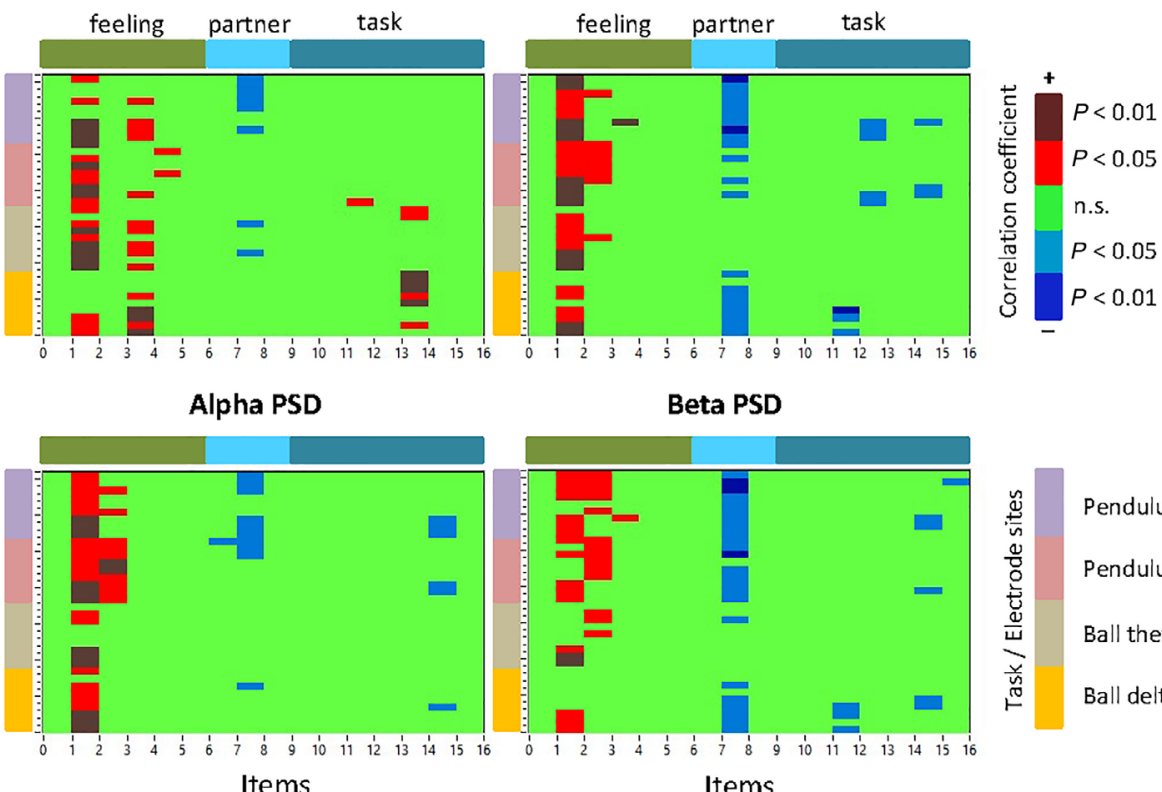
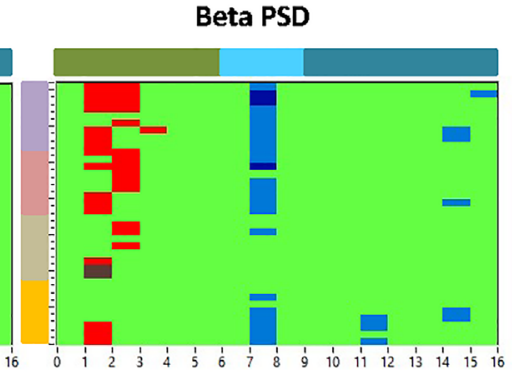

Items

\section{B}

Delta peak frequency

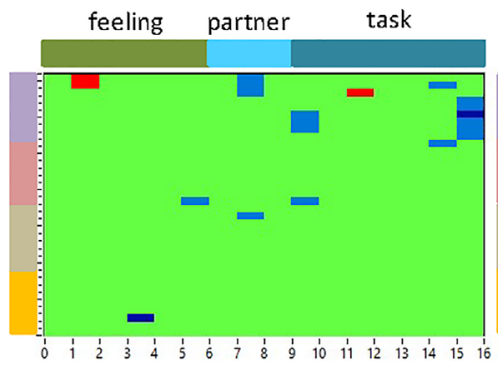

Alpha peak frequency
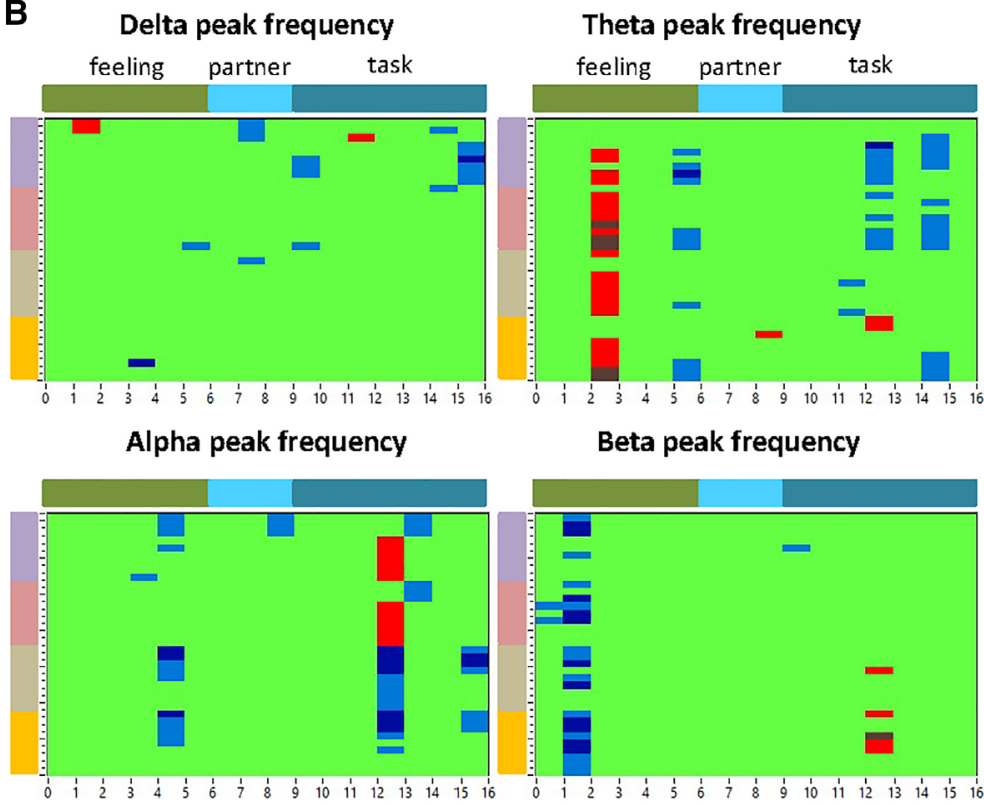

Items

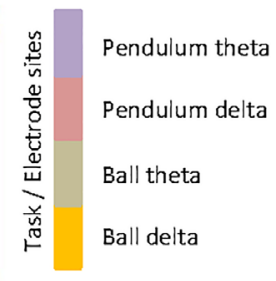

Figure 5. Correlations between the 16 postsurvey item scores and spectral power estimates in the four frequency bands. (A) Correlations between the postsurvey item scores and PSD. (B) Correlations between the postsurvey item scores and the peak frequencies. The $x$-axis represents the 16 postsurvey item scores, which are divided into three subgroups (feeling-, partner-, and task-related) as indicated by the color bars at the top of the diagrams. The $y$-axis represents the four tasks with nine electrode sites for each (see the color legend for task/electrode sites). Electrode sites are ordered as follows: FL (frontal left), FZ (mid-frontal), FR (frontal right), CL (central left), CZ (mid-central), CR (central right), PL (parietal left), PZ (mid-parietal), and PR (parietal right). The diagrams show only significant (negative and positive) correlation coefficients as indicated in the color legend. 
Table 2. ANOVA results for the intra- and interbrain coupling strengths across the four frequency bins $(2.5,5,10$, and $20 \mathrm{~Hz}$ ) in the four NFB tasks

\begin{tabular}{|c|c|c|c|c|c|c|c|c|c|c|c|c|}
\hline & \multicolumn{6}{|c|}{ Ball design } & \multicolumn{6}{|c|}{ Pendulum design } \\
\hline & \multicolumn{3}{|c|}{ Delta } & \multicolumn{3}{|c|}{ Theta } & \multicolumn{3}{|c|}{ Delta } & \multicolumn{3}{|c|}{ Theta } \\
\hline & $F$ & $P$ & $\eta^{2}$ & $F$ & $P$ & $\eta^{2}$ & $F$ & $P$ & $\eta^{2}$ & $F$ & $P$ & $\eta^{2}$ \\
\hline \multicolumn{13}{|c|}{ Intrabrain synchrony } \\
\hline \multicolumn{13}{|c|}{ Delta $(2.5 \mathrm{~Hz})$} \\
\hline $\mathrm{C}$ & 1.01 & 0.38 & 0.020 & 1.18 & 0.32 & 0.024 & 0.97 & 0.38 & 0.019 & 4.23 & 0.011 & 0.079 \\
\hline $\mathrm{C} \times \mathrm{AP}$ & 6.93 & 0.000 & 0.12 & 6.94 & 0.000 & 0.12 & 9.22 & 0.000 & 0.16 & 6.42 & 0.000 & 0.12 \\
\hline \multicolumn{13}{|c|}{ Theta $(5 \mathrm{~Hz})$} \\
\hline $\mathrm{C}$ & 0.77 & 0.50 & 0.015 & 0.69 & 0.54 & 0.014 & 0.88 & 0.41 & 0.018 & 3.95 & 0.015 & 0.075 \\
\hline $\mathrm{C} \times \mathrm{AP}$ & 10.31 & 0.000 & 0.17 & 9.21 & 0.000 & 0.16 & 13.58 & 0.000 & 0.22 & 9.61 & 0.000 & 0.16 \\
\hline \multicolumn{13}{|c|}{ Alpha $(10 \mathrm{~Hz})$} \\
\hline $\mathrm{C}$ & 3.68 & 0.020 & 0.070 & 3.96 & 0.013 & 0.075 & 2.18 & 0.12 & 0.043 & 7.66 & 0.000 & 0.14 \\
\hline $\mathrm{C} \times \mathrm{AP}$ & 22.19 & 0.000 & 0.31 & 18.25 & 0.000 & 0.27 & 20.52 & 0.000 & 0.30 & 16.41 & 0.000 & 0.25 \\
\hline \multicolumn{13}{|c|}{ Beta $(20 \mathrm{~Hz})$} \\
\hline $\mathrm{C}$ & 1.86 & 0.15 & 0.037 & 3.83 & 0.013 & 0.073 & 1.50 & 0.23 & 0.030 & 4.57 & 0.006 & 0.085 \\
\hline $\mathrm{C} \times \mathrm{AP}$ & 17.94 & 0.000 & 0.27 & 15.49 & 0.000 & 0.24 & 22.52 & 0.000 & 0.32 & 16.86 & 0.000 & 0.26 \\
\hline \multicolumn{13}{|c|}{ Interbrain synchrony } \\
\hline \multicolumn{13}{|c|}{ Delta $(2.5 \mathrm{~Hz})$} \\
\hline $\mathrm{C}$ & 12.43 & 0.000 & 0.20 & 2.07 & 0.11 & 0.41 & 2.76 & 0.058 & 0.053 & 1.24 & 0.29 & 0.025 \\
\hline $\mathrm{C} \times \mathrm{AP}$ & 0.61 & 0.63 & 0.012 & 1.73 & 0.17 & 0.034 & 3.63 & 0.014 & 0.069 & 0.49 & 0.70 & 0.010 \\
\hline \multicolumn{13}{|c|}{ Theta $(5 \mathrm{~Hz})$} \\
\hline $\mathrm{C}$ & 10.74 & 0.000 & 0.18 & 12.85 & 0.000 & 0.21 & 4.63 & 0.006 & 0.086 & 5.10 & 0.003 & 0.094 \\
\hline $\mathrm{C} \times \mathrm{AP}$ & 1.70 & 0.17 & 0.034 & 1.50 & 0.22 & 0.030 & 1.80 & 0.15 & 0.036 & 0.32 & 0.80 & 0.007 \\
\hline \multicolumn{13}{|c|}{ Alpha $(10 \mathrm{~Hz})$} \\
\hline $\mathrm{C}$ & 30.79 & 0.000 & 0.39 & 31.46 & 0.000 & 0.39 & 31.28 & 0.000 & 0.39 & 38.56 & 0.000 & 0.44 \\
\hline $\mathrm{C} \times \mathrm{AP}$ & 2.21 & 0.088 & 0.043 & 1.17 & 0.32 & 0.023 & 1.62 & 0.19 & 0.032 & 1.31 & 0.28 & 0.026 \\
\hline \multicolumn{13}{|c|}{ Beta $(20 \mathrm{~Hz})$} \\
\hline $\mathrm{C}$ & 9.21 & 0.000 & 0.16 & 4.87 & 0.008 & 0.090 & 4.75 & 0.004 & 0.088 & 8.46 & 0.000 & 0.15 \\
\hline $\mathrm{C} \times \mathrm{AP}$ & 0.85 & 0.48 & 0.017 & 0.72 & 0.56 & 0.014 & 0.83 & 0.48 & 0.017 & 1.52 & 0.19 & 0.030 \\
\hline
\end{tabular}

Bold values indicate $P<0.05$; AP, anteroposterior; C, condition; ML, mediolateral.

Figure S5 (online only), the intrabrain strengths in task conditions compared with the rest mainly decrease at frontal and central sites and increase at parietal sites.

Interbrain strengths in the delta frequency $(2.5$ $\mathrm{Hz}$ ) showed a significant main effect in the delta ball task and a significant interaction of condition $x$ anteroposterior in the delta pendulum task. Interbrain strengths in the three other frequencies (i.e., 5,10 , and $20 \mathrm{~Hz}$ ) showed a significant main effect of condition in all NFB tasks. As shown in Figure 6, the theta and beta interbrain coupling increased significantly in the NFB task conditions compared with the rest, whereas the alpha interbrain strengths decreased in the NFB tasks compared with the rest condition.

\section{Correlations of intra- and interbrain strengths} with postsurvey item scores

Correlation analyses of intra- and interbrain coupling strengths during the NFB task condition with the 16 postsurvey item scores are presented in Figure 7 . With regard to subjective feeling scores, there were negative correlations between the delta intrabrain coupling strengths and nervousness (item 3 ), and negative correlations between the intrabrain strengths at all frequencies and patience during the task (item 6). Beta intrabrain coupling correlated positively with the general test partner likeability (item 7), test partner sympathy (item 8), with the feeling that the synchronization did work overall well (item 10), and the feeling to have controlled the ball (and to some extent also the pendulum) during 
A

Delta IBS (2.5 Hz)
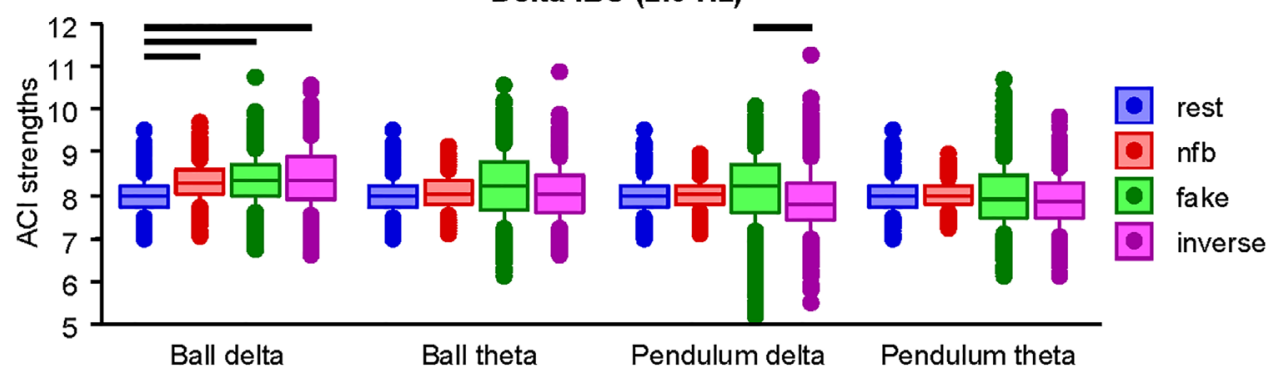

B
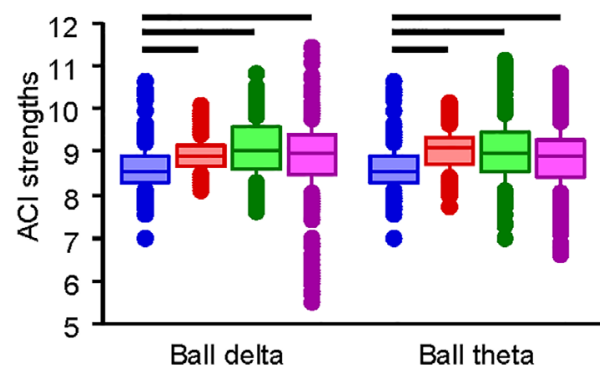

Theta IBS $(5 \mathrm{~Hz})$

C

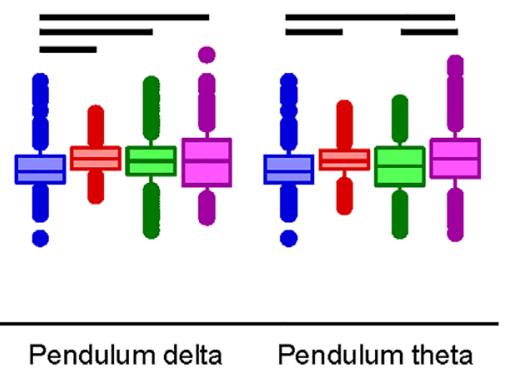

Ball theta

Pendulum delta

Alpha IBS $(10 \mathrm{~Hz})$
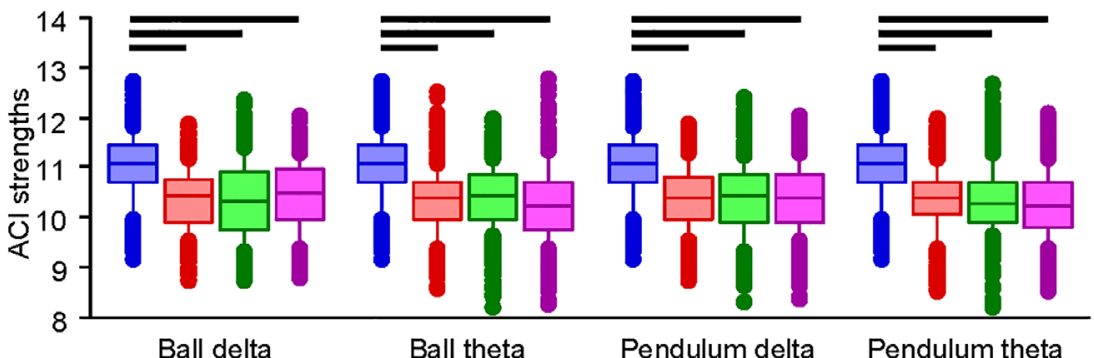

D

Ball theta

Pendulum delta

Pendulum theta

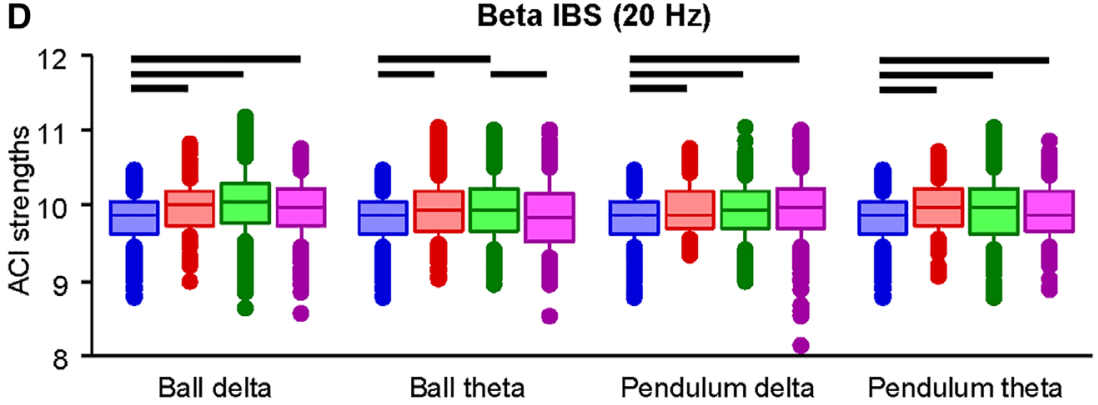

Figure 6. Box plots of the IBS changes across the four task conditions at the four frequency bins. (A) Delta IBS (2.5 Hz) across the four NFB tasks and the four task conditions. (B) Theta IBS $(5 \mathrm{~Hz})$ across the four NFB tasks and the four task conditions. (C) Alpha IBS $(10 \mathrm{~Hz})$ across the four NFB tasks and the four task conditions. (D) Beta IBS $(20 \mathrm{~Hz})$ across the four NFB tasks and the four task conditions. Box plots represent ACI strengths across the four tasks (ball delta, ball theta, pendulum delta, and pendulum theta) and the four task conditions: resting state, normal NFB, fake, and inverse task conditions. Horizontal bold lines indicate significant differences as revealed by the SNK post-hoc test $(P<0.05)$. 
A Delta intrabrain coupling Theta intrabrain coupling
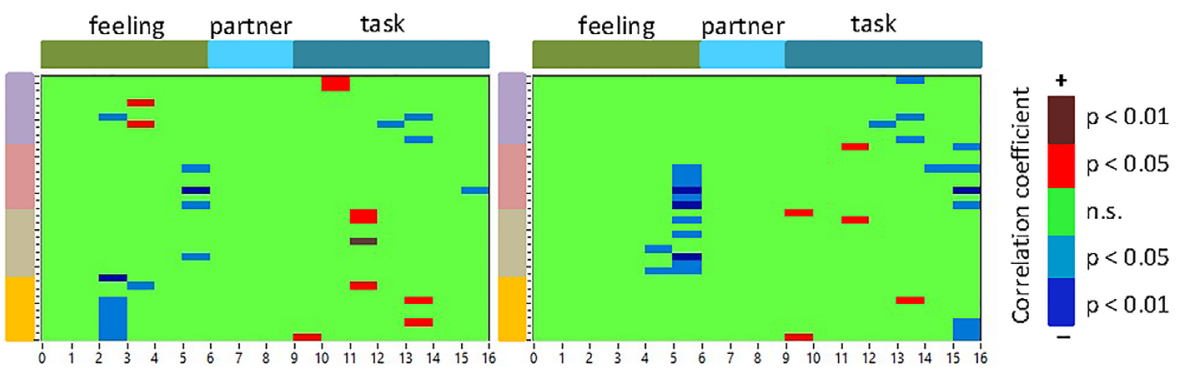

Alpha intrabrain coupling

Beta intrabrain coupling
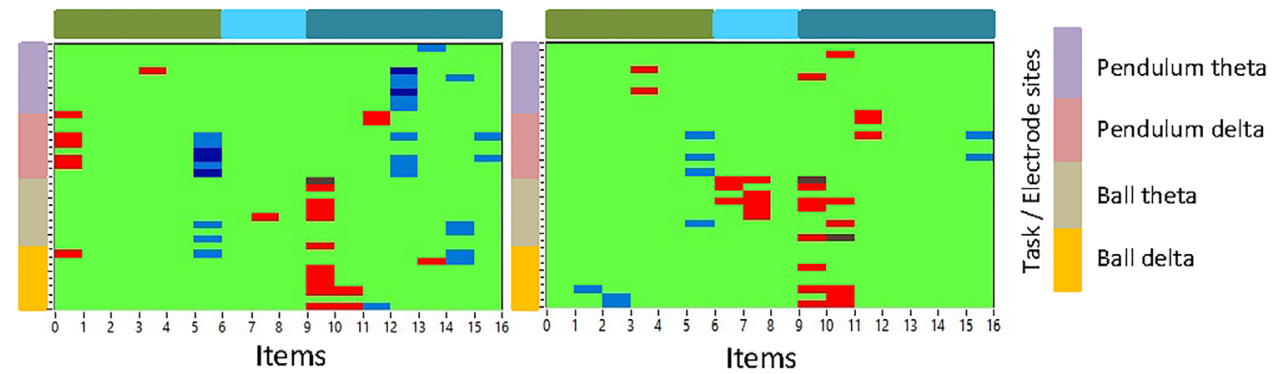

B Delta interbrain coupling

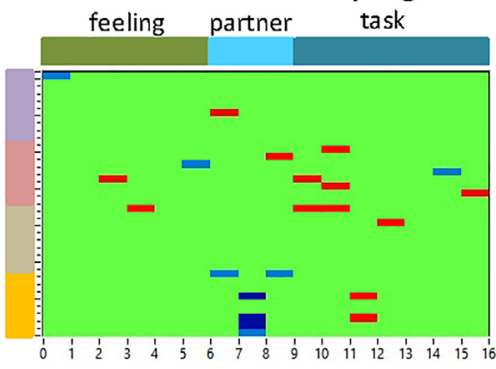

Theta interbrain coupling

feeling partner task

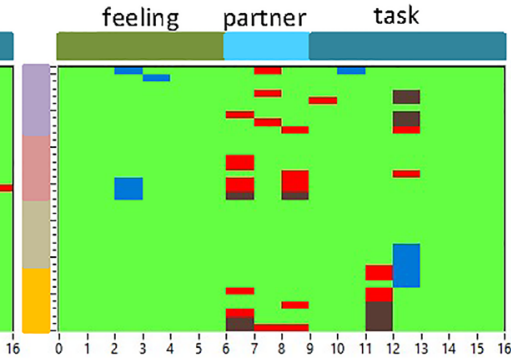

Alpha interbrain coupling

Beta interbrain coupling

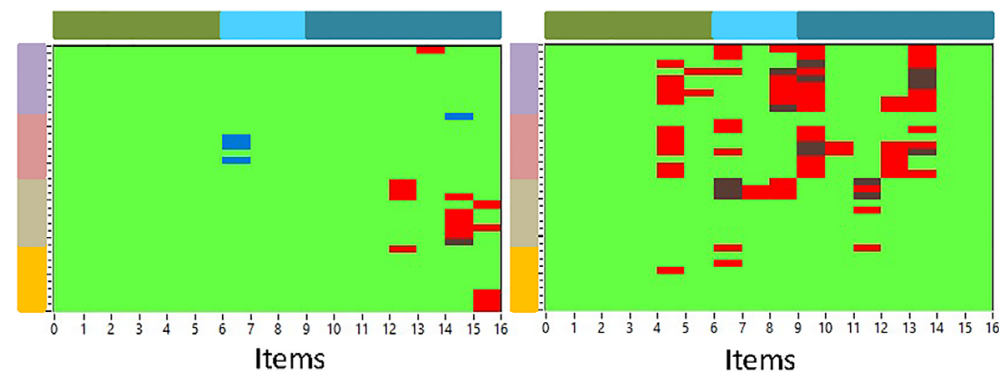

Figure 7. Correlations between the 16 postsurvey item scores and intra- and interbrain coupling strengths at the four frequencies. (A) Correlations between the 16 postsurvey item scores and intrabrain coupling strengths. (B) Correlations between the 16 postsurvey item scores and interbrain coupling strengths. The $x$-axis represents the 16 postsurvey item scores, which are divided into three subgroups (feeling-, partner-, and task-related) as indicated by the color bars at the top of the diagrams. The $y$-axis represents the four tasks with nine electrode sites for each (see the color legend for task/electrode sites). Electrode sites are ordered as follows: FL (frontal left), FZ (mid-frontal), FR (frontal right), CL (central left), CZ (mid-central), CR (central right), PL (parietal left), PZ (mid-parietal), and PR (parietal right). The diagrams show only significant (negative and positive) correlation coefficients as indicated in the color legend. 
the NFB task (item 11). During the ball task, alpha intrabrain strengths also correlated positively with the feeling that the synchronization did work overall well (item 10) and, during the pendulum task, correlated negatively with the estimated capability to influence the task through concentration (item 13) and through mental calculations (item 15).

The interbrain coupling strengths at theta and beta frequencies correlated significantly positively with the partner's likability scores (items 7-9) and the feeling that the partner could control the ball better (item 12). In addition, theta interbrain strengths correlated negatively during the ball task and positively during the pendulum task with the estimated capability to influence the task through concentration (item 13), and beta interbrain strengths correlated positively during the pendulum task with the estimated capability to influence the task through concentration and relaxation (items 13 and 14, respectively). Alpha interbrain strengths correlated significantly positively during the ball task with the estimated capability to influence the task through concentration, thoughts, and mental calculations (items 13,15 , and 16, respectively).

\section{Discussion}

In the present study, we aimed to examine the neural mechanisms of interpersonal NFB. We used two experimental designs where the subjects were able to control (1) the common state without any feedback about their own contribution to this state (ball task) and (2) the common state with the feedback about their own contribution to this state (pendulum task). These states were manipulated through the enhancement of the given feedback (fake condition) and through giving an inverted feedback (inverse condition), which distracted or confused the participants. In the ball task, the inverse feedback led to the appearance of mostly overlapped balls falsely indicating a good performance whereas, in the pendulum task with inverse feedback, the pendulums were swinging most of the time in antiphase, falsely indicating a bad performance. Thus, whereas the participants in the pendulum task were really frustrated by the negative feedback, the participants in the ball task could not understand why they were so good at times and were, thus, frustrated or disoriented by the overwhelming positive feedback. The postsurvey analysis showed some significant differences in using strategies to influence the NFB task: the generation of thoughts was used significantly more in the ball task and mental calculation was used significantly more in the pendulum task, although the last was a least used strategy as compared with other strategies.

EEG analyses revealed strong differences between the resting state and the NFB task conditions, but the differences between the NFB task conditions themselves were rather moderate or mostly absent (nonsignificant). PSD analyses showed a strong decrease of PSD in all frequency bands in the NFB task conditions compared with the rest condition. Regarding the NFB task conditions themselves, there was only a significant decrease of beta PSD in the inverse task condition compared with the normal NFB condition. This indicates that the NFB tasks led to the common desynchronization of all EEG rhythms compared with the rest. Such a decrease in spectral power (at least in the frequency ranges of $8-20 \mathrm{~Hz}$ and $35-45 \mathrm{~Hz}$ ) was also observed in the study on collective NFB mentioned above. ${ }^{46}$ An amplitude or spectral power reduction in the theta, alpha, and beta bands during the NFB task with suppression of absolute alpha amplitude has also been reported. ${ }^{55}$ As suggested by the authors and other investigators, ${ }^{56,57}$ such broadband spectral power attenuation or desynchronization could be a sign of alerting and selective attention.

Interestingly, PSD at all frequencies showed significant positive correlations with tiredness after the experiment and nervousness (except delta PSD), which were especially strong at frontocentral sites. Note that these brain sites were used for the feedback calculation and that tiredness before the experiment did not show any significant correlations. Moreover, the PSD (also in all frequency bands) correlated significantly negatively with sympathy for the test partner and also with estimated capability to influence the task. Interestingly, the latter were also found at the frontocentral sites. This indicates that a too high PSD led to tiredness and nervousness and, at the same time, lower PSD seems to positively influence the feeling of good performance or task controllability and the test partner's sympathy. There is evidence that tiredness is especially associated with low-frequency oscillations, ${ }^{58}$ and it has been suggested that alpha and beta power are related to cognitive and emotional control. ${ }^{59}$ Also, 
in the study on collective NFB, ${ }^{46}$ the decrease in broadband spectral power was associated with the ability of the participants to maintain the desired state.

Furthermore, we found that the spectral peak frequency decreases in the delta frequency range (only in the pendulum task) and especially in the theta frequency range. Importantly, the theta peak frequency converges to the task frequency of $5 \mathrm{~Hz}$. Alpha and beta peak frequencies showed only significant interactions of the factor condition with electrode sites, indicating different changes with regard to the different brain regions, whereby this was the lateral axis in the alpha frequency band and the anteroposterior axis in the beta frequency band. It should be noted that alpha and beta peak frequencies rather increased compared with the rest. Most importantly, these frequencies (i.e., alpha and beta) also showed changes within the test pairs, namely, the differences in the peak frequencies within the pairs increase compared with the rest, whereas in the NFB-task frequencies (i.e., delta and theta), there were no differences within the pairs. It seems that the adjustment of the task rhythms (i.e., delta and theta) happens due to, or is accompanied by, the divergence of the fast rhythms (alpha and beta) within the test pairs. Interestingly, the theta peak frequency correlated significantly positively with nervousness, especially at frontocentral sites, and significantly negatively with patience during the experiment and also with the estimated capability to influence the task through concentration and thoughts. The beta peak frequency correlated significantly negatively with tiredness after the experiment. This only confirms the suggestion that the decrease in theta peak frequency (and partly also in the delta peak frequency) and increase in the beta peak frequency (at least at the frontocentral brain regions) could be beneficial for the task and associated with less nervousness.

Intrabrain synchrony either shows no changes during the tasks or rather decreases compared with the resting state. Interbrain synchrony during the tasks increases compared with the rest in the theta and beta frequency ranges and partly also in the delta frequency (e.g., during the delta ball task). An increase in interbrain synchrony accompanied by a decrease in intrabrain coupling at low frequencies (e.g., delta and theta) was reported earlier with regard to interpersonal action coordination when playing guitar in a duet or a quartet. ${ }^{7,8}$ Interestingly, in the NFB study on pigeons, it has been found that both intra- and interbrain synchrony were significantly enhanced during long-term NFB training. ${ }^{49}$ Whether this was an effect of the long-term impact of the training or of the other factors remains to be seen. IBS at the alpha frequency in our study decreases significantly during the task conditions compared with the rest. Alpha and beta frequencies were not manipulated during the task, but a significant decrease of IBS in the former case and an increase in the latter indicate that these frequencies are also strongly involved. It simply shows that if the participants concentrate on a certain rhythm and change it accordingly, the whole frequency spectrum changes. It is interesting to note that some accompanying frequencies desynchronize in relation to the partner (e.g., alpha frequency) and other frequencies synchronize (e.g., beta frequency). In our opinion, this is an interesting phenomenon that needs further investigation to be better understood. Especially interesting is the strongly significant decrease in alpha IBS in the NFB task conditions compared with the rest condition. In principle, IBS during the rest condition could be regarded as a spurious synchronization, but the fact that it dropped drastically during the NFB task (regardless of the task design or task frequency) indicates that it is then more spurious than in the rest condition. In our opinion, alpha oscillations during rest are relatively stable in both participants, whereas during the task, they move apart or desynchronize in the test pair. This view is in line with the significant increase of peak frequency differences within the test pairs during the task (see Fig. S3, online only). Furthermore, it should be noted that theta and beta interbrain coupling strengths correlated mostly significantly positively with partnerand task-oriented postsurvey scores. This indicates that interbrain synchrony in these frequency bands (i.e., theta and beta), but also partly in the delta and alpha frequency bands, is relatively strongly related to the test partner's likability and estimated capability to influence the task or task frequency.

There is neurophysiological evidence that at least two large-scale neural networks that represent the self and others play a crucial role in self-related processing and social cognition. ${ }^{60}$ These are the frontoparietal mirror neuron areas connecting the 
physical self and others through motor simulation mechanisms and the cortical midline structures engaging in processing information about the self and others in more abstract, evaluative terms, ${ }^{60}$ called also the midline self-representation core. ${ }^{61}$ Interestingly, these networks are also activated for NFB interventions that are highly personalized and that attempt to alter the neural self-representation. ${ }^{61}$ The mirror neuron system, which shows similar activation during perception and execution of action $^{62}$-a process termed common coding of perception and action ${ }^{63,64}$-implies that the processing of other people's actions relies on the simulation of those actions in one's own motor system. ${ }^{65,66}$ In our NFB tasks, the participants have to mirror or predict the internal state of others and simulate their activity in the respective partner-oriented way. It has been suggested that, during an interpersonal interaction, each subject has their own forward model and corresponding neural representation of this, whereby these representations are adjusted in time. ${ }^{67}$ Moreover, they set boundary conditions for the agents to behave as a superordinate system or as a whole. ${ }^{68}$ Most importantly, our result shows that IBS is more than just an epiphenomenon of the sensorimotor response. ${ }^{4,50}$ It will be interesting to examine whether NFB based on IBS helps to improve interpersonal action coordination performance, in the sense that individuals who have learned to synchronize their brain activity by means of NFB would also improve in their ability to coordinate actions with others in real time. Experimental evidence of this sort would help to clarify the causal role of IBS for interpersonal action coordination and social interactions in general. ${ }^{50}$ Furthermore, some of the brain dynamics observed during the interpersonal NFB task were correlated to behavioral postsurvey item scores, suggesting a link to cognitive and emotional states. ${ }^{59}$ Further research is needed to better understand this association.

\section{Limitations}

The present experiment has limitations and leaves room for questions to be addressed in future research. First, we found strong differences in neural activity and synchrony during the task compared with the resting state. Using more fine-grained NFB task manipulations would lead to a more differentiated representation of NFB activity and reinforce our understanding of both NFB mechanisms and interbrain or hyperbrain interaction. Second, our analyses with regard to intra- and interbrain coupling were limited to the ACI measure reflecting the in-phase synchrony, which was important with regard to the experimental designs. Nevertheless, other types of coupling (e.g., directional or nonlinear coupling as well as multivariate coupling) are likely to provide further information about the synchronized states during interpersonal NFB.

\section{Conclusion}

Our results show that the participants were able to increase interbrain synchrony by using NFB information, especially when the interbrain coupling was fed back at the theta frequency. Apart from the interbrain coupling, other oscillatory activities (e.g., PSD, peak amplitude and peak frequency, and intrabrain coupling) changed during the task compared with the rest condition. Moreover, all the measures showed specific correlations with the subjective postsurvey item scores, indicating specific relations of neural oscillatory activity to subjective feeling and appraisal. Finally, this study shows that hyperscanning with IBS feedback seems to be an important tool to examine interbrain oscillatory coupling and performance that provides important information about neural mechanisms of social interaction and collective behavior. It thus opens new perspectives for the causal assessment of IBS.

\section{Acknowledgments}

This research was supported by the Max Planck Society. The authors are grateful to Oksana Berhe, Vivien Choupurian, Shiva Motlagh, Katrin Müller, and Alireza Tarikhi for technical assistance and carrying out the experimental part of the study. The authors thank Christel Fraser for language assistance, and Berndt Wischnewski and Ioanna Michopoulou for software assistance.

Open access funding enabled and organized by Projekt DEAL.

\section{Author contributions}

V.M., D.P., M.A.M., and U.L. designed the research. V.M., D.P., and M.A.M. performed the research. V.M. analyzed the data. V.M., D.P., and M.A.M. wrote the paper. All authors read and approved the final manuscript. 


\section{Supporting information}

Additional supporting information may be found in the online version of this article.

Table S1. Post-survey items.

Table S2. ANOVA results for the PSD across the four frequency bands in the four NFB tasks.

Table S3. ANOVA results for the peak amplitude across the four frequency bands in the four NFB tasks.

Table S4. ANOVA results for the peak frequency across the four frequency bands in the four NFB tasks.

Table S5. ANOVA results for the peak frequency differences within the test pairs across the four frequency bands in the four NFB tasks.

Table S6. ANOVA results for the intrabrain coupling strengths across the four frequency bins (2.5, 5,10 , and $20 \mathrm{~Hz}$ ) in the four NFB tasks.

Table S7. ANOVA results for the interbrain coupling strengths across the four frequency bins (2.5, 5,10 , and $20 \mathrm{~Hz}$ ) in the four NFB tasks.

Figure S1. PSD changes along the anteroposterior axis across the task conditions at the four frequency bins.

Figure S2. Box plots of the peak amplitude changes across the task conditions in the four frequency bands.

Figure S3. The peak frequency and peak frequency difference changes within the test pairs across the task conditions in the alpha and beta frequency bands.

Figure S4. Intrabrain synchrony changes across the task conditions at the four frequency bins.

Figure S5. Intrabrain synchrony changes along the anteroposterior axis across the task conditions at the four frequency bins.

\section{Videos S1-S6.}

\section{Competing interests}

The authors declare no competing interests.

\section{References}

1. Baltes, P. \& U. Staudinger. 1996. Interactive minds in a lifespan perspective: prologue. In Interactive Minds: Life-Span
Perspectives on the Social Foundation of Cognition. P.B. Baltes \& U.M. Staudinger, Eds.: 1-32. New York: Cambridge University Press.

2. Gallese, V. 2003. The manifold nature of interpersonal relations: the quest for a common mechanism. Philos. Trans. $R$. Soc. Lond. B. Biol. Sci. 358: 517-528.

3. Dumas, G., J. Nadel, R. Soussignan, et al. 2010. Interbrain synchronization during social interaction. PLoS One 5. e12166.

4. Lindenberger, U., S.-C. Li, W. Gruber, et al. 2009. Brains swinging in concert: cortical phase synchronization while playing guitar. BMC Neurosci. 10: 22.

5. Balconi, M. \& M.E. Vanutelli. 2017. Cooperation and competition with hyperscanning methods: review and future application to emotion domain. Front. Comput. Neurosci. 11: $1-6$.

6. Czeszumski, A., S. Eustergerling, A. Lang, et al. 2020. Hyperscanning: a valid method to study neural inter-brain underpinnings of social interaction. Front. Hum. Neurosci. 14: 39.

7. Müller, V., J. Sänger \& U. Lindenberger. 2018. Hyperbrain network properties of guitarists playing in quartet. Ann. N.Y. Acad. Sci. 1423: 198-210.

8. Müller, V., J. Sänger \& U. Lindenberger. 2013. Intra- and inter-brain synchronization during musical improvisation on the guitar. PLoS One 8. e73852.

9. De Vico Fallani, F., V. Nicosia, R. Sinatra, et al. 2010. Defecting or not defecting: how to "read" human behavior during cooperative games by EEG measurements. PLoS One 5. e14187.

10. Goldstein, P., I. Weissman-Fogel, G. Dumas, et al. 2018. Brain-to-brain coupling during handholding is associated with pain reduction. Proc. Natl. Acad. Sci. USA 115: E2528E2537.

11. Müller, V. \& U. Lindenberger. 2014. Hyper-brain networks support romantic kissing in humans. PLoS One 9. e112080.

12. Müller, V. \& U. Lindenberger. 2019. Dynamic orchestration of brains and instruments during free guitar improvisation. Front. Integr. Neurosci. 13: 50.

13. Sänger, J., V. Müller \& U. Lindenberger. 2012. Intra- and interbrain synchronization and network properties when playing guitar in duets. Front. Hum. Neurosci. 6: 312.

14. Frith, C.D. \& U. Frith. 2007. Social cognition in humans. Curr. Biol. 17: R724-R32.

15. Hari, R. \& M.V. Kujala. 2009. Brain basis of human social interaction: from concepts to brain imaging. Physiol. Rev. 89: 453-479.

16. Elmer, S. \& L. Jäncke. 2014. Intracerebral functional connectivity-guided neurofeedback as a putative rehabilitative intervention for ameliorating auditory-related dysfunctions. Front. Psychol. 5: 1-7.

17. Emmert, K., R. Kopel, J. Sulzer, et al. 2016. Meta-analysis of real-time fMRI neurofeedback studies using individual participant data: how is brain regulation mediated? Neuroimage 124: 806-812.

18. Imperatori, C., G. Marca, N. Amoroso, et al. 2017. Alpha/theta neurofeedback increases mentalization and default mode network connectivity in a non-clinical sample. Brain Topogr. 30: 822-831. 
19. Lecomte, G. \& J. Juhel. 2011. The effects of neurofeedback training on memory performance in elderly subjects. Psychology 2: 846-852.

20. Birbaumer, N. \& L.G. Cohen. 2007. Brain-computer interfaces: communication and restoration of movement in paralysis. J. Physiol. 579: 621-636.

21. Lubar, J.F., M.O. Swartwood, J.N. Swartwood, et al. 1995. Evaluation of the effectiveness of EEG neurofeedback training for ADHD in a clinical setting as measured by changes in T.O.V.A. scores, behavioral ratings, and WISC-R performance. Biofeedback Self Regul. 20: 83-99.

22. Rockstroh, B., T. Elbert, N. Birbaumer, et al. 1993. Cortical self-regulation in patients with epilepsies. Epilepsy Res. 14: 63-72.

23. Chapin, T.J. \& L.A. Russell-Chapin. 2014. Neurotherapy and Neurofeedback: Brain-Based Treatment for Psychological and Behavioral Problems. London: Routledge.

24. Demos, J.N. 2005. Getting Started with Neurofeedback. New York: W. W. Norton \& Company.

25. Brunner, C., R. Scherer, B. Graimann, et al. 2006. Online control of a brain-computer interface using phase synchronization. IEEE Trans. Biomed. Eng. 53: 2501-2506.

26. Gonuguntla, V., Y. Wang \& K.C. Veluvolu. 2013. Phase synchrony in subject-specific reactive band of EEG for classification of motor imagery tasks. In Proceedings of the Annual International Conference of the IEEE Engineering in Medicine and Biology Society EMBS, 2784-2787.

27. Gysels, E. \& P. Celka. 2004. Phase synchronization for the recognition of mental tasks in a brain-computer interface. IEEE Trans. Neural Syst. Rehabil. Eng. 12: 406-415.

28. Sacchet, M.D., J. Mellinger, R. Sitaram, et al. 2012. Volitional control of neuromagnetic coherence. Front. Neurosci. 6: 189.

29. Wei, Q., Y. Wang, X. Gao, et al. 2007. Amplitude and phase coupling measures for feature extraction in an EEG-based brain-computer interface. J. Neural Eng. 4: 120-129.

30. Birbaumer, N., N. Ghanayim, T. Hinterberger, et al. 1999. A spelling device for the paralysed. Nature 398: 297-298.

31. Birbaumer, N., A. Kübler, N. Ghanayim, et al. 2000. The thought translation device (TTD) for completely paralyzed patients. IEEE Trans. Rehabil. Eng. 8: 190-193.

32. Kotchoubey, B., D. Schneider, H. Schleichert, et al. 1996. Self-regulation of slow cortical potentials in epilepsy: a retrial with analysis of influencing factors. Epilepsy Res. 25: 269-276.

33. Kotchoubey, B., S. Busch, U. Strehl, et al. 1999. Changes in EEG power spectra during biofeedback of slow cortical potentials in epilepsy. Appl. Psychophysiol. Biofeedback 24: 213-233.

34. Ramot, M., S. Kimmich, J. Gonzalez-Castillo, et al. 2017. Direct modulation of aberrant brain network connectivity through real-time neurofeedback. eLife 6. e28974.

35. Strehl, U., B. Kotchoubey, T. Trevorrow, et al. 2005. Predictors of seizure reduction after self-regulation of slow cortical potentials as a treatment of drug-resistant epilepsy. Epilepsy Behav. 6: 156-166.

36. Jeunet, C., L. Tonin, L. Albert, et al. 2020. Uncovering EEG correlates of covert attention in soccer goalkeepers: towards innovative sport training procedures. Sci. Rep. 10: 1705.
37. Linden, M., T. Habib \& V. Radojevic. 1996. A controlled study of the effects of EEG biofeedback on cognition and behavior of children with attention deficit disorder and learning disabilities. Biofeedback Self Regul. 21: 3549.

38. Lubar, J.O. \& J.F. Lubar. 1984. Electroencephalographic biofeedback of SMR and beta for treatment of attention deficit disorders in a clinical setting. Biofeedback Self Regul. 9: 1-23.

39. Lubar, J.F. 1991. Discourse on the development of EEG diagnostics and biofeedback for attention-deficit/hyperactivity disorders. Biofeedback Self Regul. 16: 201-225.

40. Wang, Q., O. Sourina \& M.K. Nguyen. 2011. Fractal dimension based neurofeedback in serious games. Vis. Comput. 27: 299-309.

41. Vernon, D., T. Egner, N. Cooper, et al. 2003. The effect of training distinct neurofeedback protocols on aspects of cognitive performance. Int. J. Psychophysiol. 47: 75-85.

42. Rasey, H.W., J.F. Lubar, A. Mcintyre, et al. 1996. EEG biofeedback for the enhancement of attentional processing in normal college students. J. Neurother. 1: 15-21.

43. Beatty, J., A. Greenberg, W.P. Deibler, et al. 1974. Operant control of occipital theta rhythm affects performance in a radar monitoring task. Science 183: 871-873.

44. Belluomo, P., M. Bucolo, L. Fortuna, et al. 2011. Robot control through brain computer interface for patterns generation. Complex Syst. 20: 1031-1034.

45. Egner, T. \& J.H. Gruzelier. 2003. Ecological validity of neurofeedback: modulation of slow wave EEG enhances musical performance. Neuroreport 14: 1221-1224.

46. Kovacevic, N., P. Ritter, W. Tays, et al. 2015. 'My Virtual Dream': collective neurofeedback in an immersive art environment. PLoS One 10. e0130129.

47. Duan, L., W.J. Liu, R.N. Dai, et al. 2013. Cross-brain neurofeedback: scientific concept and experimental platform. PLoS One 8. e64590.

48. Zhang, R. \& X. Zhao. 2018. A cross-brain interaction platform based on neurofeedback using electroencephalogram. In Lecture Notes in Artificial Intelligence. D.D. Schmorrow \& C.M. Fidopiastis, Eds.: 220-230. Cham: Springer.

49. Yang, L., M. Li, L. Yang, et al. 2020. Functional connectivity changes in the intra- and inter-brain during the construction of the multi-brain network of pigeons. Brain Res. Bull. 161: $147-157$.

50. Novembre, G. \& G.D. Iannetti. 2021. Hyperscanning alone cannot prove causality. Multibrain stimulation can. Trends Cogn. Sci. 25: 96-99.

51. Müller, V. \& U. Lindenberger. 2011. Cardiac and respiratory patterns synchronize between persons during choir singing. PLoS One 6. e24893.

52. Baker, J.M., N. Liu, X. Cui, et al. 2016. Sex differences in neural and behavioral signatures of cooperation revealed by fNIRS hyperscanning. Sci. Rep. 6: 26492.

53. Reysen, S. 2005. Construction of a new scale: the Reysen likeability scale. Soc. Behav. Pers. 33: 201-208.

54. Vigário, R.N. 1997. Extraction of ocular artefacts from EEG using independent component analysis. Electroencephalogr. Clin. Neurophysiol. 103: 395-404. 
55. Ros, T., J. Théberge, P.A. Frewen, et al. 2013. Mind over chatter: plastic up-regulation of the fMRI salience network directly after EEG neurofeedback. Neuroimage 65: 324-335.

56. Fan, J., J. Byrne, M.S. Worden, et al. 2007. The relation of brain oscillations to attentional networks. J. Neurosci. 27: 6197-6206.

57. Friese, U., J. Daume, F. Göschl, et al. 2016. Oscillatory brain activity during multisensory attention reflects activation, disinhibition, and cognitive control. Sci. Rep. 6: 32775.

58. Wyczesany, M., J. Kaiser \& A.M.L. Coenen. 2008. Subjective mood estimation co-varies with spectral power EEG characteristics. Acta Neurobiol. Exp. (Wars) 68: 180-192.

59. Ray, W.J. \& H.W. Cole. 1985. EEG alpha activity reflects attentional demands, and beta activity reflects emotional and cognitive processes. Science 228: 750-752.

60. Uddin, L.Q., M. Iacoboni, C. Lange, et al. 2007. The self and social cognition: the role of cortical midline structures and mirror neurons. Trends Cogn. Sci. 11: 153-157.

61. Ioannides, A.A. 2018. Neurofeedback and the neural representation of self: lessons from awake state and sleep. Front. Hum. Neurosci. 12: 142.
62. Schmidt, R.C., P. Fitzpatrick, R. Caron, et al. 2011. Understanding social motor coordination. Hum. Mov. Sci. 30: 834845.

63. Novembre, G., L.F. Ticini, S. Schütz-Bosbach, et al. 2014. Motor simulation and the coordination of self and other in real-time joint action. Soc. Cogn. Affect. Neurosci. 9: 10621068.

64. Sebanz, N. \& G. Knoblich. 2009. Prediction in joint action: what, when, and where. Top. Cogn. Sci. 1: 353-367.

65. Wolpert, D.M., K. Doya \& M. Kawato. 2003. A unifying computational framework for motor control and social interaction. Philos. Trans. R. Soc. B Biol. Sci. 358: 593602.

66. Wolpert, D.M. \& Z. Ghahramani. 2000. Computational principles of movement neuroscience. Nat. Neurosci. 3: 1212-1217.

67. Sänger, J., U. Lindenberger \& V. Müller. 2011. Interactive brains, social minds. Commun. Integr. Biol. 4: 655-663.

68. Müller, V., J.A.M. Delius \& U. Lindenberger. 2018. Complex networks emerging during choir singing. Ann. N.Y. Acad. Sci. 1431: 85-101. 